\title{
Schweizerische Akademie für Qualität in der Medizin: SAOM
}

\section{Roxane Küblera, Esther Kraft ${ }^{b}$, Christoph Bosshard ${ }^{c}$}

a MA, Operative Leiterin der Schweizerischen Akademie für Qualität in der Medizin SAQM der FMH; 'b lic. rer. oec., Leiterin Abteilung Daten, Demographie und Qualität DDQ der FMH; ${ }^{\circ}$ Dr. med., Vizepräsident der FMH, Departementsverantwortlicher Daten, Demographie und Qualität

Seit bald fünf Jahren setzt sich die SAQM der FMH für Qualitätsarbeit in der Medizin ein. Im Fokus stehen hierbei Projekte, die sich dem Patientennutzen, der Qualitätssicherung sowie deren Weiterentwicklung widmen. Zudem bietet die SAQM u.a. für Ärzte- und Partnerorganisationen unterschiedliche Dienstleistungen im Bereich der medizinischen Qualität an.

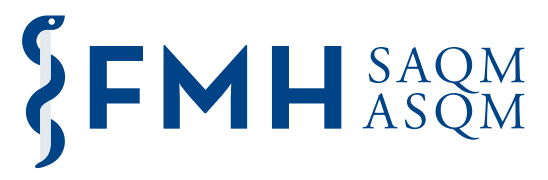

2012 gründete die FMH die Schweizerische Akademie für Qualität in der Medizin SAQM. Als eine Organisation der Schweizer Ärzteschaft ist sie zuständig für alle Belange der Qualität in der Medizin. Die SAQM engagiert sich für eine kontinuierliche Qualitätsentwicklung. Sie

- fördert alle Aspekte der medizinischen Qualitätsarbeit mit Nutzen für Patienten, Angehörige und Ärzte;

- unterstützt die Entwicklung einer Qualitätskultur und setzt sich für einen hohen Standard von Qualitätsprojekten sowie bei der Erhebung, Auswertung und Interpretation von Qualitätsdaten ein;

- engagiert sich für den Zusammenhalt der Ärzteschaft beim Thema Qualität und unterstützt die Vernetzung von Qualitätsaktivitäten der verschiedenen Fachgesellschaften;

- setzt sich für die verstärkte Verankerung der Qualitätsfragen in der Aus-, Weiter- und Fortbildung der Ärzte ein;

- bezieht die Partner des Gesundheitswesens mit ein;

- ist Ansprechpartnerin und Koordinatorin für die ärztlichen Aspekte der Begleit- und Versorgungsforschung sowie Verhandlungspartnerin bei Qualitätsthemen auf nationaler Ebene;

- kommuniziert zu Qualitätsthemen innerhalb der Ärzteschaft und nach aussen und repräsentiert sie zum Thema Qualität. liche interessierten Parteien / Interessensgruppen sind eingeladen, in den Dialog Qualität Einsitz zu nehmen. Ihre Bewerbungen sind unter der Mail-Adresse (saqm[at]fmh.ch) willkommen.

\section{Qualitätsprojekte der SAQM 2016}

Die SAQM entwickelt die medizinische Qualitätsarbeit weiter - durch eigene Projekte und indem sie externe Projekte unterstützt und begleitet. 2016 hat sie unter anderem an folgenden Projekten gearbeitet (Auswahl):

(1) Sektorenübergreifender Behandlungspfad Kolorektalkarzinom

Die Fortschritte in der Behandlung von Krankheiten und die Spezialisierung haben zur Folge, dass immer mehr Spezialisten unterschiedlicher Fachgebiete in die Behandlungskette involviert sind. Für die Patienten bedeutet dies, dass sie über einen Zeitraum hinweg entweder sequenziell oder parallel von verschiedenen medizinischen Fachpersonen behandelt werden.

Gemeinsam mit 20 medizinischen Fachgesellschaften und nichtärztlichen Berufsgruppen hat die SAQM in einem Pilotprojekt am Beispiel des Kolorektalkarzinoms einen sektoren- und berufsgruppenübergreifenden Behandlungspfad entwickelt.

(2) Gemeinsame Empfehlungen zum Aufbau und Betrieb von gesundheitsbezogenen Registern Register mit verlässlichen Daten werden im Gesundheitswesen immer wichtiger und mit den wachsenden Datenmengen zahlreicher. Um zur Qualitätssicherung beizutragen, haben die Organisationen $\mathrm{FMH}, \mathrm{ANQ}^{1}, \mathrm{H}^{2}{ }^{2}, \mathrm{SAMW}^{3}$ und unimedsuisse $\mathrm{ge}^{4}$ meinsam Empfehlungen für den Aufbau und Betrieb von gesundheitsbezogenen Registern heraus- 
gegeben. Diese enthalten Mindeststandards, unter anderem zum Datenschutz und zur Datenqualität. Die Empfehlungen bieten des Weiteren Grundlagen, um die Qualität laufender sowie künftiger Register zu prüfen (www.saqm.ch $\rightarrow$ Publikationen $\rightarrow$ Empfehlungen Register).

(3) Interprofessionelle Peer-Review-Verfahren

Hierbei geht es um ein national einheitliches interprofessionelles Peer-Review-Verfahren, das sich bei statistischen Auffälligkeiten durchführen lässt: Die Spitäler analysieren gemeinsam mit externen Peers (Fachkollegen) Patientenakten, um Behandlungsabläufe zu verbessern. Der interprofessionelle Ansatz erlaubt es, neben spital- und fachübergreifenden Lösungen zur Steigerung von Qualität und Patientensicherheit auch berufsübergreifende Verbesserungen zu erarbeiten.

(4) Ideelle und finanzielle Projektunterstützung der Stiftung für Patientensicherheit

Als Gründungsmitglied unterstützt die FMH die Stiftung für Patientensicherheit ideell und finanziell. In den Jahren 2015/2016 stand dabei die Patientensicherheit bei der Versorgung psychisch erkrankter Menschen im Zentrum. Die Stiftung Patientensicherheit Schweiz führte das Projekt «Patientensicherheit in der psychiatrischen Versorgung - Bestandsaufnahme und Aktionsplan» durch. Der Aktionsplan zeigt auf, was die nächsten Schritte zur Verbesserung der Patientensicherheit in der psychiatrischen Versorgung in den kommenden Jahren sein müssen. Um für das Thema zu sensibilisieren und tatsächlich Wirkung zu erzielen, hat die Stiftung den Aktionsplan 2016 (http://www.patienten sicherheit.ch/de/themen/Identifikation-von-Risiken/ Patientensicherheit-in-der-psychiatrischen-Versorgung.html) schweizweit in drei Landessprachen bei wichtigen Berufsverbänden, Fachgesellschaften und Weiterbildungsorganen bekannt gemacht.

(5) Grundlagen- und Positionspapier zum Thema "Overuse» und zu «Choosing Wisely"

Die SAQM erarbeitet regelmässig Grundlagenpapiere zu verschiedenen Qualitätsthemen, ausgehend vom aktuellen Stand der Wissenschaft. Sie werden regelmässig in der Schweizerischen Ärztezeitung veröffentlicht. Die FMH nimmt auf Basis dieser Grundlagenpapiere mit den «Positionen der FMH» öffentlich Stellung zum Thema. 2016 sind Grundlagen- und Positionspapiere zu den Themen «Overuse - unnötige Behandlungen als Qualitätsproblem» (http:// www.fmh.ch/files/pdf17/SAEZ-04424.pdf) und «Choosing Wisely - für weniger unnötige Leistungen» entstanden (http://www.fmh.ch/files/pdf18/ SAEZ-Grundlagenpapier_Choosing_Wisely.pdf).
Detailliertere Informationen zu diesen und zu weiteren Projekten der SAQM finden Sie auf unserer Website: www.saqm.ch $\rightarrow$ Qualitätsprojekte.

\section{Qualitäts-Charta SAQM}

Die Qualitäts-Charta hält politische Grundsätze für Qualität in der Medizin schriftlich fest. Sie wurde in einem zweijährigen Prozess von den Delegierten des Forum Qualität der SAQM entwickelt und richtet sich an Ärzteorganisationen. Mit der freiwilligen Unterzeichnung der Charta bekennen sich diese zu Kooperation und Vernetzung in Qualitätsfragen in der Schweiz. Die Charta baut auf drei Pfeilern auf: erstens Transparenz, d.h. Chartamitglieder machen ärztliche Aktivitäten zur Förderung der Qualität in der Medizin sichtbar. Zweitens Verbindlichkeit, d.h. sie entwickeln eine Qualitätsstrategie und evaluieren die Arbeiten regelmässig in einem Qualitätsbericht. Sowie drittens Nachhaltigkeit, d.h. Chartamitglieder stellen Qualitätsentwicklung sicher.

2016 wurde die Charta in den in der Ärztekammer vertretenen Organisationen breit vernehmlasst und verabschiedet. Seit September 2016 ist die Unterzeichnung der Charta möglich. Bereits sind 67 Ärzteorganisationen der Charta beigetreten und engagieren sich gemeinsam für die Qualitätsarbeit in der Medizin. Für sie gilt es nun, die Inhalte der Qualitäts-Charta mit einer Qualitätsstrategie und einem ersten Qualitätsbericht umzusetzen. Die Unterzeichnung der Qualitäts-Charta ist und bleibt freiwillig und ist weiterhin jederzeit möglich. Kontaktieren Sie uns dazu am besten per E-Mail via saqm[at] fmh.ch.

\section{SAQM-Dienstleistungen für Qualität}

Die Dienstleistungen der SAOM stehen den Ärzte- und Partnerorganisationen sowie auch Einzelpersonen und Dritten zur Verfügung. In folgenden Bereichen kann eine Unterstützung durch die SAOM beantragt werden:

- Vernetzung und Koordination in medizinischen Qualitätsfragen

- Fachliche Unterstützung zu Qualitätsfragen

- Eingabe neuer Themen

Punktuelle finanzielle Unterstützung

Seit der Gründung der SAQM vor vier Jahren sind über $60 \mathrm{An}$ träge eingereicht und bearbeitet worden. Weitere Informationen erhalten Sie hierzu auf der SAQM-Website unter der Rubrik "Unterstützung" (www.saqm.ch $\rightarrow$ Dienstleistungen $\rightarrow$ Unter stützung).

\section{SAQM-Newsletter}

Der SAQM-Newsletter informiert Sie ca. 5- bis 6-mal jährlich per E-Mail über Projekte, Arbeiten und neueste Publikationen der SAQM. Wir freuen uns über Ihr Interesse und Ihre Anmeldung via saqm[at]fmh.ch 
Kantonale Ärztegesellschaften

Aargauischer Ärzteverband

Ärztegesellschaft Baselland

Ärztegesellschaft des Kantons Luzern

Ärztegesellschaft des Kantons Schwyz

Ärztegesellschaft des Kantons St. Gallen

Ärztegesellschaft des Kantons Zürich

Ärztegesellschaft Thurgau

Association des Médecins du canton de Genève

Gesellschaft der Ärztinnen und Ärzte des Kantons Solothurn

Ordine dei Medici del Cantone Ticino

Société de médecine du canton de Fribourg

Société Neuchâteloise de Médecine

Unterwaldner Ärztegesellschaft

Walliser Ärztegesellschaft

\section{Fachgesellschaften}

Schweiz. Fachgesellschaft für Tropen- und Reisemedizin FMH

Schweiz. Gesellschaft für Allergologie und Immunologie

Schweiz. Gesellschaft für Allgemeine Innere Medizin

Schweiz. Gesellschaft für Anästhesiologie und Reanimation

Schweiz. Gesellschaft für Arbeitsmedizin

Schweiz. Gesellschaft für Chirurgie

Schweiz. Gesellschaft für Dermatologie und Venerologie

Schweiz. Gesellschaft für Gastroenterologie

Schweiz. Gesellschaft für Gefässchirurgie

Schweiz. Gesellschaft für Gynäkologie und Geburtshilfe

Schweiz. Gesellschaft für Hämatologie

Schweiz. Gesellschaft für Handchirurgie

Schweiz. Gesellschaft für Herz- und thorakale Gefässchirurgie

Schweiz. Gesellschaft für Infektiologie

Schweiz. Gesellschaft für Intensivmedizin

Schweiz. Gesellschaft für Kardiologie

Schweiz. Gesellschaft für Kinder- und Jugendpsychiatrie und -psychotherapie

Schweiz. Gesellschaft für klinische Pharmakologie und Toxikologie

Schweiz. Gesellschaft für Medizinische Genetik

Schweiz. Gesellschaft für Medizinische Onkologie

Schweiz. Gesellschaft für Neurochirurgie

Schweiz. Gesellschaft für Nuklearmedizin

Schweiz. Gesellschaft für ORL, Hals- und Gesichtschirurgie

Schweiz. Gesellschaft für Pädiatrie

Schweiz. Gesellschaft für Pathologie

Schweiz. Gesellschaft für Plastisch-Rekonstruktive und Ästhetische Chirurgie

Schweiz. Gesellschaft für Pneumologie

Schweiz. Gesellschaft für Psychiatrie und Psychotherapie

Schweiz. Gesellschaft für Radiologie

Schweiz. Gesellschaft für Radio-Onkologie

Schweiz. Gesellschaft für Rechtsmedizin

Schweiz. Gesellschaft für Rheumatologie

Schweiz. Gesellschaft für Thoraxchirurgie

Schweiz. Gesellschaft für Urologie

Schweiz. Neurologische Gesellschaft

Schweiz. Ophthalmologische Gesellschaft 
Swiss Orthopaedics

Swiss Society for Interventional Pain Management

Dachverbände, Basisorganisationen und weitere medizinische Verbindungen

$\mathrm{fmCh}$

FMP

FMPP

Haus- und Kinderärzte Schweiz

Kollegium für Hausarztmedizin

medical women switzerland

Réseau Delta

Schweiz. Ärztegesellschaft für Manuelle Medizin

Schweizerische Belegärzte-Vereinigung

Schweizerische Gesellschaft der Vertrauens- und Versicherungsärzte

Schweizerische Gesellschaft für Notfall- und Rettungsmedizin

Schweizerische Gesellschaft für Traumatologie und Versicherungsmedizin

SFSM

Union Schweizerischer komplementärmedizinischer Ärzteorganisationen

VSAO

Fotos der Erst-Unterzeichnung an der Ärztekammer im Oktober 2016, SAQM-Vorlagen für eine Qualitätsstrategie und für einen Qualitätsbericht sowie weitere Informationen zur Qualitäts-Charta SAQM sind auf der Webseite der SAQM zugänglich (www.saqm.ch $\rightarrow$ Qualitäts-Charta).

Diese 67 Ärzteorganisationen haben die QualitätsCharta SAQM unterzeichnet (Stand: 8. Mai 2017):

\section{Ausblick 2017}

Auch in diesem Jahr macht sich die SAQM im Dienste der Ärzteschaft mit spannenden Projekten und Themenschwerpunkten für die Qualität in der Medizin stark.

- So begleitet die SAQM die Umsetzung der QualitätsCharta. Unter anderem bietet sie hierfür einen "Quality Support» an. Damit unterstützt sie die unterzeichnenden Organisationen darin, ihre Qualitätsstrategie zu entwickeln und über ihre qualitätsfördernden Aktivitäten zu berichten. Denn viele Inhalte existieren bereits. Bei manchen Organisationen geht es nun noch darum, strukturiert explizit zu machen, was bereits geleistet wird.

- Ein weiterer Schwerpunkt in diesem Jahr stellt das Pilotprojekt "Patient Centered Outcome Registry" (PCOR) dar. Im Zentrum stehen dabei die Bedürfnisse des einzelnen Patienten im Kontext der Begleitumstände und Komorbiditäten. Gelingt es mit- tels der gemeinsam definierten Abklärungs- und Behandlungsschritte, das gemeinsam definierte Ziel $\mathrm{zu}$ erreichen? Dieses Instrument soll schliesslich Best-Practice-Empfehlungen ermöglichen, welche auf Alltagserfahrungen basieren, und so eine wertvolle Ergänzung zu den evidenzbasierten Guidelines bilden.

- Die Plattform Qualitäts-Initiativen wird überarbeitet. Damit soll ein aktueller Überblick über die seitens der Organisationen der FMH empfohlenen Aktivitäten ermöglicht werden.

- All diese sowie die bereits laufenden oder abgeschlossenen Projekte bilden zusammen mit der Vernetzungsarbeit der SAQM die Grundlage, auf welcher die FMH sich auch beim Gesetzgebungsprozess im Rahmen der Änderung KVV für die «Stärkung von Qualität und Wirtschaftlichkeit» einbringt.

Das grosse Spektrum der Projekte und Tätigkeiten der SAQM zeigt, dass sie in einem breiten Feld unseres Gesundheitswesens aktiv ist und als Ansprechpartner für Qualitätsthemen in der Medizin wahrgenommen wird. Im Zentrum der Arbeiten steht der Mehrwert, den kompetente Qualitätsarbeit für Patienten, Angehörige und Ärzte schafft.

Weiterführende Informationen: www.saqm.ch

Abbildung Logo SAQM

Quelle: FMH 